\title{
Beitrag zur Kenntniss einiger seltener Erden
}

\author{
von
}

\section{Ludwig Haber.}

tus dem k. k. III. chem. Universitätslaboratorium des Prof. Ed. Lippmann.

(Mit 1 Textfigur.)

(Vorgelegt in der Sitzung am 8. Juli 1897.)

In Folge der stets steigenden Verwendung des Thoriums, sowie der mit demselben verwandten Erden, wie Cer, Lanthan, Didym und Zirkon, anderseits in Folge der durch die Auffindung und Ausnützung grösserer Lagerstätten eingetretenen Preisreduction haben diese Verbindungen auch in weiteren Kreisen grössere Beachtung gefunden und sind häufiger Gegenstand wissenschaftlicher Forschung geworden.

Die Eigenschaften der Salze dieser Elemente weichen von denjenigen ihrer Verwandten, als welche in gewisser Hinsicht Aluminium, Beryllium, Zinn u. s. w. zu betrachten sind, theilweise bedeutend $a b$, während sie unter einander in mancher Beziehung grosse Ähnlichkeit zeigen, so in Bezug auf das Verhalten zu Oxalsäure, Schwefelsäure u. s. w. Thorium und Zirkonium zeigen wiederum grössere Neigung zur Bildung: von basischen Salzen als die Elemente der Cer-Gruppe und werden erstere durch Alkalien vor den letzteren gefällt.

Ich habe gefunden, dass die seltenen Elemente unter oft ganz einfachen Verhältnissen mit einigen organischen Säuren oder Salzen derselben Verbindungen einzugehen im Stande sind, die meistens einen basischen Charakter, also geringeren Säuregehalt als die normal zusammengesetzten Salze besitzen. Zirkonium und Thorium geben ausserdem mit Chromsäure oder Kaliumbichromat sehr charakteristische Salze, welche Cer, Lanthan, Didym u. s. w. durchaus nicht zeigen. 
Einige der so erhaltenen Producte sind durch schwere Löslichkeit und Krystallisationsvermögen ausgezeichnet, so dass ihre Abscheidung und Reindarstellung eine sehr leichte ist.

Im Nachfolgenden soll das Verhalten der seltenen Erden zu Chromsäure und Kaliumbichromat, dann zu Natriumacetat und -Formiat, ferner zu den gewöhnlichen organischen Säuren, wie Weinsäure, Citronen- und Äpfelsäure, besprochen werden.

\section{Verhalten gegen Chromsäure und Bichromate. ${ }^{1}$}

Wird eine nur wenig freie Säure enthaltende Lösung von Thoriumnitrat $\left[\mathrm{Th}\left(\mathrm{NO}_{3}\right)_{4}\right]$ bei hinreichender Verdünnung einige Zeit mit Chromsäure gekocht, so bildet sich ein prächtig krystallinischer, orangegelb gefärbter Niederschlag von Thoriumchromat, welchen man noch leichter und in grösserer Menge erhält, wenn man statt der Chromsäure Natrium- oder Kaliumbichromat anwendet. Man wäscht das zu Boden fallende Salz ein- bis zweimal durch Decantation mit heissem Wasser und filtrirt. Der Niederschlag ist in Wasser fast unlöslich.

Getrocknet und unter dem Mikroskop betrachtet, erweist. sich derselbe als ein Conglomerat von sehr deutlich ausgebildeten, spitzwinkeligen, flachen rhombischen Tafeln von folgender Gestalt:

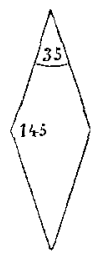

In mässig concentrirten Mineralsäuren löst sich das Thoriumchromat. Seine Bildung erfolgt nach der Reaction: $\mathrm{Th}\left(\mathrm{NO}_{3}\right)_{4}+\mathrm{Na}_{2} \mathrm{Cr}_{2} \mathrm{O}_{7}+\mathrm{H}_{2} \mathrm{O}=\mathrm{Th}\left(\mathrm{CrO}_{4}\right)_{2}+2 \mathrm{NaNO}_{3}+2 \mathrm{HNO}_{3}$.

Die frei gewordene Salpetersäure löst einen Theil des gebildeten Thoriumchromates, und bei Überschuss von Natriumbichromat wird die entsprechende Menge Chromsäure frei, die ebenfalls theilweise lösend wirkt.

1 Neutrales Chromat gibt bekannterweise sofort eine Fällung, die so erhaltenen Verbindungen zeigen aber kein krystallinisches Aussehen. 
Die Analyse ${ }^{1}$ des lufttrockenen Salzes ergibt:

I. $0.5640 \mathrm{~g}$ gaben bei $130-140^{\circ} 0.0578 \mathrm{~g}$ Wasser, $0.1643 \mathrm{~g}$ $\mathrm{Cr}_{2} \mathrm{O}_{3}$ und $0 \cdot 2836 g$ Th $\mathrm{O}_{2}$.

II. $0.4067 \mathrm{~g}$ gaben, in verdünnter Salpetersäure gelöst, mit Oxalsäure gerade gefällt und geglüht, $0.2040 \mathrm{~g} \mathrm{ThO}_{\mathrm{a}}$. Die Chromsäure wird im Filtrat in gewöhnlicher Weise mit unterschwefligsaurem Natrium titrirt. Verbrauch an $1 / 10^{-N o r m a l l o ̈ s u n g ~} 46 \cdot 5 \mathrm{~cm}^{3}$.

In 100 Theilen:

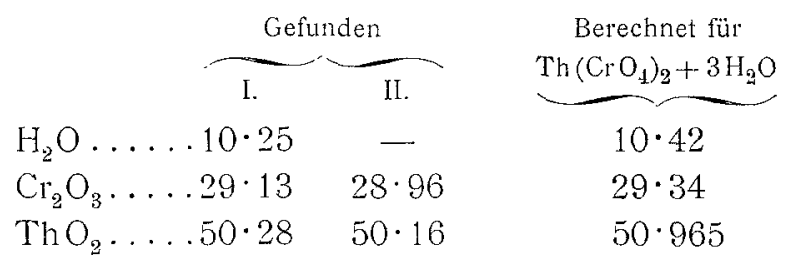

Cer, Lanthan und Didym bilden, wie schon erwähnt, unter diesen Verhältnissen, d. h. bei Gegenwart von freier Säure, keine Chromate; hingegen wird das Zirkonium durch freie Chromsäure leicht gefä1lt. Dieser Niederschlag ist flockig und ähnlich orangegelb gefärbt wie das Thoriumchromat; in verdünnten Säuren ist derselbe kaum löslich.

Die Analyse dieses Zirkonchromates ergab Zahlen, die auf eine basische Zusammensetzung des Salzes schliessen lassen, da ein weit höherer Gehalt an Zirkondioxyd $\left(\mathrm{ZrO}_{2}\right)$ gefunden wurde, als bei einer normal zusammengesetzten Verbindung zu erwarten war. Es liegt die Vermuthung nahe, dass die Chromsäure durch Wasser entfernt wird, worauf die Analyse von Producten verschiedener Darstellung schliessen lässt. Eine gänzliche Entfernung der Chromsäure war dagegen auch durch anhaltendes Kochen mit Wasser nicht zu erzielen.

1 Dieselbe wird am besten in der Weise ausgeführt, dass man mit Natrium- und Kaliumcarbonat schmilzt und die Schmelze in verdünnte Salzsäure aufnimmt, wobei unlösliche weisse Thorerde $\left(\mathrm{Th}_{2}\right)$ zurückbleibt, während das Chrom als Kaliumchromat $\left(\mathrm{K}_{2} \mathrm{CrO}_{4}\right)$ in Lösung geht und in bekannter Weise bestimmt wird. 
I. $0 \cdot 4903 \mathrm{~g}$ bis zur Gewichtsconstanz getrockneter Substanz gaben $0.3233 g \mathrm{ZrO}_{2}$ und $0.0741 g \mathrm{Cr}_{2} \mathrm{O}_{3}$.

II. $0 \cdot 6504 \mathrm{~g}$ bis zur Gewichtsconstanz getrockneter Substanz gaben $0.4277 \mathrm{~g} \mathrm{ZrO}_{2}$ und $0.1005 \mathrm{~g} \mathrm{Cr}_{2} \mathrm{O}_{3}$.

III. $0.7117 \mathrm{~g}$ anderer Darstellung bis zur Gewichtsconstanz getrockneter Substanz gabęn $0.4362 \mathrm{~g} \mathrm{ZrO}_{2}$ und $0.1380 \mathrm{~g}$ $\mathrm{Cr}_{2} \mathrm{O}_{3}$.

In 100 Theilen:

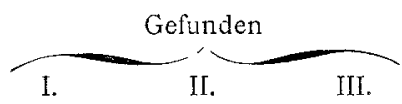

$$
\begin{array}{lll}
\mathrm{ZrO}_{2} \ldots \ldots .65 \cdot 94 & 65 \cdot 76 & 61 \cdot 29 \\
\mathrm{Cr}_{2} \mathrm{O}_{3} \ldots \ldots .15 \cdot 11 & 15 \cdot 45 & 19 \cdot 39
\end{array}
$$

Die Summe von $\mathrm{ZrO}_{2}$ und $\mathrm{Cr}_{2} \mathrm{O}_{3}$ ist constant; Salze verschiedener Darstellung sind aber, wie erwähnt, nicht gleich zusammengesetzt.

\section{Verhalten gegen Natriumacetat.}

Auf Zusatz von Natriumacetat zur Lösung eines Thoriumsalzes bleibt letztere lange Zeit, vollkommen klar. Erst beim Kochen tritt ein feinkörniger, schwerer, weisser Niederschlag auf, der sich rasch absetzt und die Eigenschaft besitzt, aus sehr verdünnten Lösungen $(0 \cdot 1 \%)$ erst nach längerem Kochen in Form von Flocken auszufallen.

Nach dem Filtriren, Auswaschen u. s. w. stellt dieser Niederschlag ein blendend weisses Krystallpulver dar, welches sich in Wasser fast gar nicht, in concentrirten Mineralsäuren und Eisessig hingegen leicht löst.

Die Analyse ergab, ${ }^{\bullet}$ dass ein basisches Thoriumacetat vorliegt:

I. $0.5361 \mathrm{~g}$ Substanz gaben, bei $130^{\circ}$ getrocknet, $0.0236 \mathrm{~g}$ Wasser und bei anhaltendem Glühen $0.3499 \mathrm{~g} \mathrm{Th}_{2}$. Das gebildete Thoroxyd ist anfänglich schwarz und nimmt oft erst nach dem Erhitzen im Gebläse eine rein weisse Farbe an.

II. $0 \cdot 4300 \mathrm{~g}$ Substanz ergaben geglüht $0 \cdot 2798 g \mathrm{gh} \mathrm{T}_{2}$. 
III. $0.9470 \mathrm{~g}$ Substanz verbrauchten nach der Destillation mit verdünnter Schwefelsäure zur Neutralisation an $2 / 10^{-}$Normalkalilauge $23 \cdot 2 \mathrm{~cm}^{3}$.

In 100 Theilen:

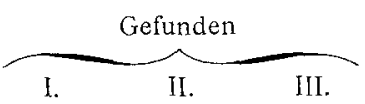

Wasser... 4.402 - -

$\mathrm{ThO}_{2} \ldots .65 \cdot 27 \quad 65 \cdot 07 \quad-$

$\mathrm{CH}_{3}-\mathrm{COOH}-29 \cdot 40$

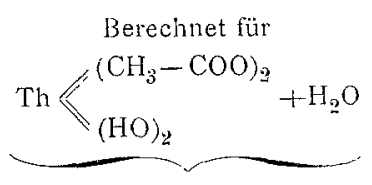

$4 \cdot 48$

$65 \cdot 67$

Cer, Lanthan und Didym werden auch nach längerem Kochen mit Natriumacetat nicht gefällt. Die Bildung des oben besprochenen Thoriumacetates erfolgt auch bei einem Gehalt von $10-15 \%$ an Ceritverbindungen; dieselbe gibt uns demnach ein Mittel an die Hand, cerfreies Thoroxyd zu erhalten, was für die Praxis von Bedeutung ist.

Durch Lösen des Thoriumacetates in einer Mineralsäure und Eindampfen der Lösung kann man leicht verlässlich reines Thoriumsalz erhalten und durch eventuelle Wiederholung dieses Processes (etwa Verwandlung des Acetates in das Nitrat und nochmalige Fällung mit essigsaurem Natrium) auch stark mit Cer verunreinigtes Thorium rein darstellen. Dasselbe erwies sich wie eine spectral-analytische Untersuchung ergab als didymfrei.

Wird eine Zirkonlösung mit Natriumacetat gekocht, so bildet sich ein weisser, flockig-gelatinöser Niederschlag, der hartnäckig Wasser zurückhält und bei vorsichtigem Trocknen schliesslich in eine amorphe Masse von harzartiger Consistenz übergeht.

Im Filtrat lässt sich mit den gewöhnlichen Reagentien kein Zirkonium nachweisen.

Das bis zur Gewichtsconstanz getrocknete Salz ergab bei der Analyse Zahlen, die gleichfalls einen sehr hohen Gehalt an Zirkondioxyd $\left(\mathrm{ZrO}_{2}\right)$, nämlich $67 \cdot 624 \%$ zeigten. Um die Brauchbarkeit der Fällungsmethode durch essigsaures Natrium 
in Bezug auf die quantitative Abscheidung des Zirkons aus Lösungen seiner Salze zu erproben, wurde eine Zirkonnitratlösung $\left[\mathrm{Zr}\left(\mathrm{NO}_{3}\right)_{1}\right]$ von bekanntem Gehalt dargestellt $\left(1 \mathrm{~cm}^{3}=\right.$ $0.0296 \mathrm{~g} \mathrm{ZrO}_{2}$ ).

$10 \mathrm{~cm}^{3}$ dieser Lösung ergaben nach dem Fällen mit essigsaurem Natrium, heissem Auswaschen u. s. w. und schliesslichem Glühen des basischen Zirkonacetat $0.2932 \mathrm{~g}, \mathrm{ZrO}_{2}$ was $99.05 \%$ entspricht. Daraus ist ersichtlich, dass die Abscheidung des Zirkons durch essigsaures Natrium in Form von basischem Acetat beinahe quantitativ verläuft und zur Analyse verwendet werden kann.

Das dem Thoriumacetat entsprechende Thoriumformiat konnte bei analoger Darstellung nicht in vollkommen reinem Zustande erhalten werden, und für diese Thatsache stimmen auch die Analysen von Producten verschiedener Darstellung. Auch bildet sich das Salz schwerer; es fällt erst bei starker Verdünnung und anhaltendem Kochen als ein sehr schwerer, weisser, feinkörniger Niederschlag aus, welcher, wie die mikroskopische Betrachtung zeigt, aus ganz distincten, schön ausgebildeten, durchsichtigen Hexaëdern besteht.

Analyse:

$0.6167 g$ wasserfreier Substanz ergaben bei der Verbrennung mit Kupferoxydasbest $0.132 \mathrm{~g} \mathrm{CO}_{2}, 0.0628 \mathrm{~g} \mathrm{H}_{2} \mathrm{O}$ und $0.4795 \mathrm{~g} \mathrm{ThO}_{2}$.

In 100 Theilen:

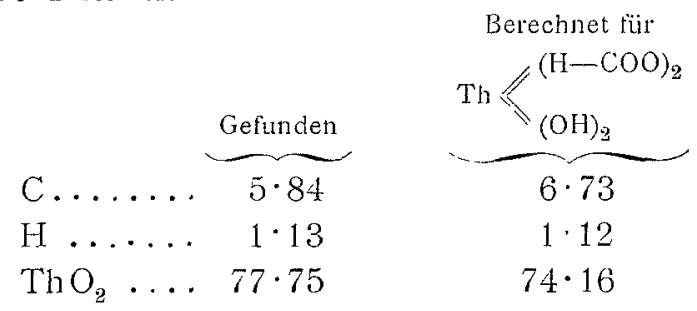

Die Zahlen, welche das Verhältniss zwischen Thorium und Ameisensäure ausdrücken, liegen zwischen den Werthen $1: 2$ und $2: 3$ (etwa $3: 5$ ). 
Im Gegensatze zu Natriumacetat bildet das Natriumformiat mit Cer, Lanthan, Didym bei Siedehitze und hinreichender Concentration prächtig krystallisirte Salze, die, ausgeschieden und gewaschen, in heissem und kaltem Wasser sehr schwer löslich sind und normale Constitution besitzen.

Ich habe mich speciell mit dem Cerformiat eingehender beschäftigt, da dasselbe stets bei noch so genauer Analyse einen geringern Gehalt an Cerdioxyd ergab als der Theorie entspricht. Die jüngsten Untersuchungen von Schützenberger und Wyruboff ${ }^{1}$ über das Atomgewicht des Cers bewogen mich, auf die Sache näher einzugehen. Es handelte sich vor Allem um die verlässliche Reindarstellung eines Cersalzes, zu welchem Zwecke ich den schon von Pop $\mathrm{p}^{2}$ angegebenen Weg, wenn auch in modificirter Weise, einschlug.

Ich fällte in der Hitze mit Natriumacetat und oxydirte mit Bromwasser (nicht, wie Popp, durch Einleiten von Chlor), löste in wenig Salzsäure und fällte die stark verdünnte Lösung abermals mit essigsaurem Natrium und Brom. Dieser Vorgang wurde oftmals (bis siebenmal) wiederholt, das Cerchlorid schliesslich mit Ameisensäure, ein anderer Theil mit Oxalsäure gefällt und dann geglüht.

Es ergaben sich für das Cer bedeutend kleinere, untereinander differirende Atomgewichte, und behalte ich mir vor, auf diesen Gegenstand nach Abschluss der diesbezüglichen Untersuchungen zurückzukommen.

Versetzt man eine Zirkonsalzlösung mit ameisensaurem Natrium, so erfolgt schon bei gelindem Erwärmen die Bildung eines flockigen, weissen, voluminösen Niederschlages und nahezu vollständige Fällung des Zirkoniums.

Die Analysen dieser Verbindung führten zu einem analogen Resultat wie beim Zirkonacetat.

I. $0 \cdot 4440 \mathrm{~g}$ der bis zur Gewichtsconstanz getrockneten Substanz ergaben $0 \cdot 3390 \mathrm{~g} \mathrm{ZrO}_{2}$.

1 Schützenberger, C. r. Bd. 124, Ni. 10 vom 8. März 1897. Die Abhandlung Wyruboff's ist noch neueren Datums, nur aus einer Mittheilung in der Chemikerzeitung bekannt.

2 A. 131, 359. 
II. $0 \cdot 6153$ g getrocknete Substanz verbrauchten (nach Lie be n) $30.5 \mathrm{~cm}^{3} \quad \mathrm{KMnO}_{4}$-Lösung $\left(1 \mathrm{~cm}^{3}=0.0087 \dot{7} \mathrm{~g} \mathrm{KMnO}_{4}\right)$.

In 100 Theilen:

$$
\begin{array}{cc}
\mathrm{ZrO}_{2} \ldots \ldots \ldots \ldots & \underbrace{\text { Gefunden }}_{76 \cdot 35} \\
\mathrm{H}-\mathrm{COOH} \ldots \ldots & 19 \cdot 00
\end{array}
$$

Dieses sowie die anderen besprochenen Zirkonsalze entsprechen einem Gemenge von $x \cdot Z \cdot{ }_{r} \cdot \stackrel{I}{\Sigma}_{4}+y \cdot Z r(O H)_{4}$, wobei $\stackrel{\text { I. }}{\Sigma}$ das einwerthige Säureradical bedeutet.

\section{Weinsäureverbindungen.}

Zum Unterschiede von den vorgenannten Salzen, die durch Fällung mit freier Säure nicht gebildet werden, wirken Weinsäure, Citronensäure und Äpfelsäure als solche direct fällend ein. Über ein weinsaures Thorium wurde von Cleve ${ }^{1}$ berichtet, welcher dasselbe durch Erhitzen von Thoriumchloridlösung und Weinsäure erhielt. Seine Analyse führt zu der Formel:

$$
\begin{gathered}
\mathrm{Th}_{3}\left(\mathrm{C}_{4} \mathrm{H}_{4} \mathrm{O}_{6}\right)_{4}(\mathrm{HO})_{4}+5 \mathrm{H}_{2} \mathrm{O} \\
\text { (gefunden } \mathrm{C} 13 \cdot 45, \mathrm{H} 2 \cdot 46, \mathrm{ThO}_{2} 54 \cdot 63 \text { ). }
\end{gathered}
$$

Ich habe das Salz in analoger Weise dargestellt; es ist ein flockiger weisser Niederschlag, der langsam trocknet und schwer einen bestimmten Krystallwassergehalt ermitteln lässt. Er zeigt in dieser Beziehung eine gewisse Ähnlichkeit mit gefälltem Thonerdehydrat $\left[\mathrm{Al}(\mathrm{OH})_{3}\right]$.

Deshalb wurde erst das bis zur Gewichtsconstanz getrocknete Salz zur Analyse verwendet; diese ergibt:

I. $0.5064 g$ gaben nach dem Glühen $0.323 g \mathrm{Th} \mathrm{O}_{2}$.

II. $0.5350 \mathrm{~g}$ gaben bei der Verbrennung mit Kupferoxydasbest $0.2660 \mathrm{~g} \mathrm{CO}_{2}$ und $0.0672 \mathrm{~g} \mathrm{H}_{2} \mathrm{O}$. Bei der Verbrennung blieben zurück: $0 \cdot 3303 \mathrm{~g} \mathrm{ThO}_{2}$. III. $0.6247 \mathrm{~g}$ gaben $0.3068 \mathrm{~g} \mathrm{CO}_{2}$ und $0.0712 \mathrm{~g} \mathrm{H}_{2} \mathrm{O}$.

1 Bulletin de la société chimique de Paris, vol. 21, p. 115. 
In 100 Theilen:

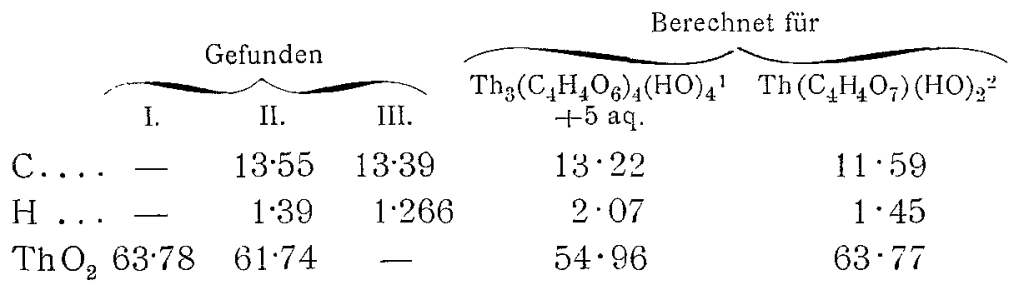

Wenn man diese von Cleve angenommene Constitution als richtig annimmt, so findet man, wie aus obiger Analyse ersichtlich, einen zu grossen Thorerdegehalt. Bei der von mir supponirten, an zweiter Stelle erwähnten einfacheren Constitutionsformel stimmt der Thoriumgehalt in einem Falle ganz scharf; allerdings finde ich zu viel Kohlenstoff.

Während Cer, Lanthan und Didym durch Weinsäure nicht fallen, verhält sich das Zirkonium ganz ähnlich wie in den früher besprochenen Fällen. Auf Zusatz von Weinsäure $z u$ einer heissen Lösung von Zirkoniumnitrat entsteht sofort wiederum ein weisser, voluminöser, flockiger Niederschlag, welcher im Überschusse des Fällungsmittels löslich ist, schwer filtrirt und leicht trüb durch das Filter geht. Beim Trocknen wird auch dieser ursprünglich gelatinöse Niederschlag hart und pulverisirbar. Durch die Analyse wurde gefunden, dass auch hier kein constant zusammengesetztes Salz vorliegt (s. o.).

\section{Citronensaures Thorium.}

Das Thoriumcitrat wurde nach derselben Methode wie das vorerwähnte Salz mit Citronensäure erhalten und stellt weisse Flocken dar, die ein dichteres Gefüge besitzen als das weinsaure Thorium. Bei längerer Einwirkung von kaltem Wasser löst sich das Salz theilweise und fällt bein Erhitzen wieder aus.

$$
\begin{aligned}
& 1(\mathrm{OH})_{4} \equiv \mathrm{Th}_{3} \stackrel{\text { XII. }}{\mathrm{T}}\left[\mathrm{COO}-\mathrm{C}_{2} \mathrm{H}_{2}(\mathrm{OH})_{2}-\mathrm{COO}\right]_{1}+5 \mathrm{aq} . \\
& \text { IV. } \\
& =(\mathrm{OH})_{2} \mathrm{Th}\left[\mathrm{COO}-\mathrm{C}_{2} \mathrm{H}_{2}(\mathrm{OH})_{2}-\mathrm{COO}\right] .
\end{aligned}
$$


Analyse:

I. $0 \cdot 5000 \mathrm{~g}$ bis zur Gewichtsconstanz getrockneter Substanz. gaben bei der Verbrennung mit Kupferoxydasbest $0.3005 \mathrm{~g}$ $\mathrm{CO}_{2}$ und $0.0695 \mathrm{~g} \mathrm{H}_{2} \mathrm{O}$. Bei der Verbrennung bleiben als Rückstand $0 \cdot 2993 \mathrm{~g} \mathrm{ThO}_{2}$.

II. $0 \cdot 4833 \mathrm{~g}$ Substanz anderer Darstellung ergaben beim Glühen $0 \cdot 2898 g$ Th $\mathrm{O}_{2}$.

In 100 Theilen:

\begin{tabular}{|c|c|c|}
\hline \multirow{2}{*}{\multicolumn{2}{|c|}{ Gefunden }} & \multirow{2}{*}{$\begin{array}{r}\begin{array}{l}\text { Berechnet für } \\
\text { Th. } \mathrm{C}_{6} \mathrm{H}_{6} \mathrm{O}_{8}\end{array} \\
\end{array}$} \\
\hline I. & & \\
\hline$\ldots 16 \cdot 39$ & - & $16 \cdot 44$ \\
\hline$\ldots 1 \cdot 54$ & - & $1 \cdot 37$ \\
\hline$\ldots .59 \cdot 86$ & $59 \cdot 96$ & $60 \cdot 27$ \\
\hline
\end{tabular}

Obige empirische Formel entspricht folgender Constitutionsformel:

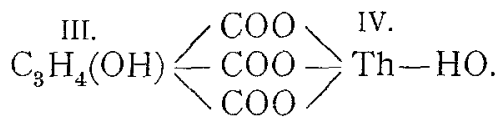

\section{Äpfelsaures Thorium.}

Dasselbe fällt durch Zusatz von freier Äpfelsäure, $\mathrm{COOH}-\mathrm{C}_{2} \mathrm{H}_{3}(\mathrm{OH})-\mathrm{COOH}$, nach längerem Kochen als weisser körnig-krystallinischer Niederschlag, der sich rasch absetzt und in Wasser fast unlöslich ist.

Analyse:

$0.5450 \mathrm{~g}$ bis zur Gewichtsconstanz getrockneter Substanz gaben $0.3060 \mathrm{~g} \mathrm{CO}_{2}$ und $0.0700 \mathrm{~g} \mathrm{H}_{2} \mathrm{O}$. Rückstand nach der Verbrennung $0.3277 g \mathrm{ThO}_{2}$.

In 100 Theilen:

\begin{tabular}{|c|c|c|}
\hline & $\underbrace{\text { Gefunden }}$ & $\begin{array}{c}\text { Berechnet für } \\
\mathrm{Th}_{2}\left(\mathrm{C}_{1} \mathrm{H}_{4} \mathrm{O}_{5}\right)_{3}(\mathrm{OH})_{2}\end{array}$ \\
\hline$C \ldots \ldots$ & $15 \cdot 31$ & $16 \cdot 11$ \\
\hline $\mathrm{H} \ldots \ldots$ & $1 \cdot 127$ & $1 \cdot 61$ \\
\hline${ }^{\prime} \mathrm{HhO}_{2} \ldots$ & 6013 & $59 \cdot 06$ \\
\hline
\end{tabular}


Als Structurformel finden wir:

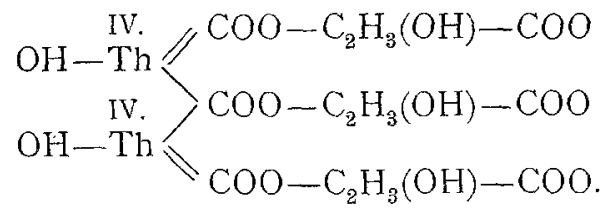

Die Eigenschaften des citronensauren und äpfelsauren Zirkoniums, soferne es mit freier Säure gefällt ist und daher basischen Charakter besitzt, sind denjenigen der früher behandelten Salze ganz analog; auch diese Verbindungen zeigen keine constante Zusammensetzung.

Wie aus den mitgetheilten Thatsachen erhellt, zeigen die oben besprochenen Elemente gegenüber den angewandten Reagentien verschiedenes Verhalten: Zirkonium wild fast vollständig gefällt (quantitative Abscheidung), was beim Thorium meist nicht der Fall ist; die Metalle der Ceritgruppe fallen entweder gar nicht oder unter Bildung von Verbindungen anderer Constitution.

Charakteristisch für Thorium und Zirkonium ist die Bildung basischer Salze mit den organischen Säuren; doch zeigen hier wiederum die Thoriumverbindungen eine constante Zusammensetzung, woraluf übrigens schon ibr Aussehen schliessen lässt, während das Zirkonium äusserst leicht verseifbare, gelatinöse Niederschläge liefert, die sich in ihrer Zusammensetzung bei fortwährender Behandlung mit Wasser immer mehr dem Hydrat nähern.

Die benützten Präparate wurden theilweise von der chemischen Fabrik von Dr. Theodor Schuchardt Görlitz in vorzüglicher Reinheit bezogen, theilweise von mir selbst aus dem Monazitsande dargeslellt. Die zu analysirenden Endproducte habe ich durch oftmalige Wiederholung der Operationen gereinigt.

Zur leichteren Übersicht der Reactionen füge ich umstehende Tabelle bei. 


\begin{tabular}{|c|c|c|c|}
\hline 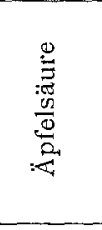 & 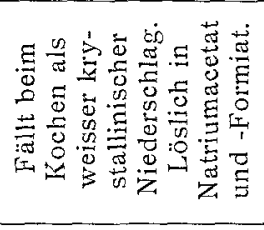 & 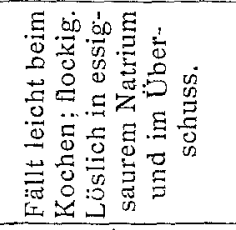 & 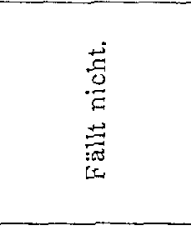 \\
\hline 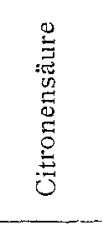 & 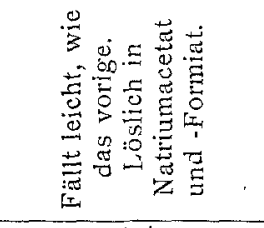 & 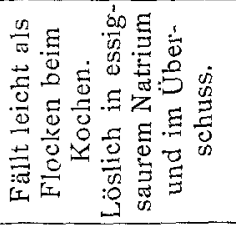 & 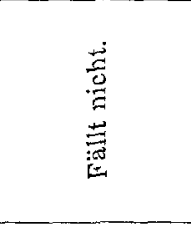 \\
\hline 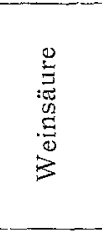 & 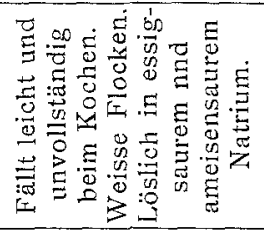 & 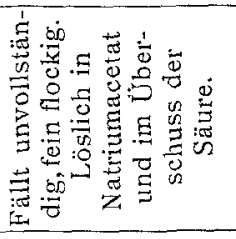 & 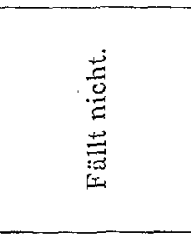 \\
\hline 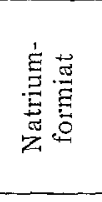 & 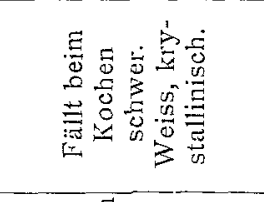 & 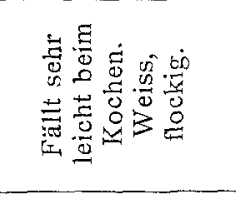 & 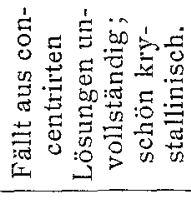 \\
\hline 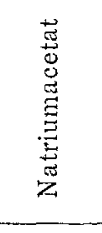 & 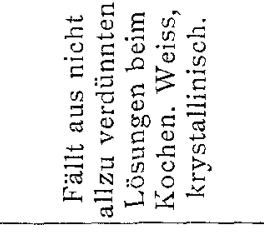 & 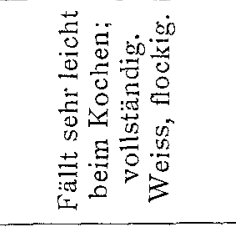 & 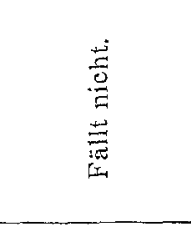 \\
\hline 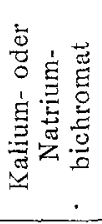 & 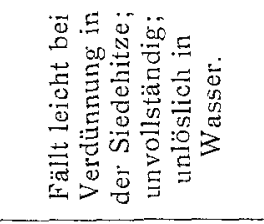 & 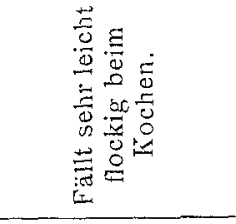 & - \\
\hline 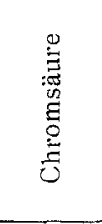 & 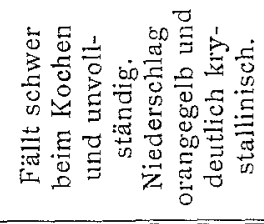 & 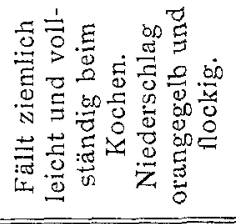 & 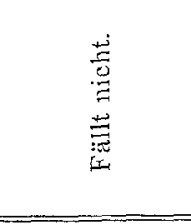 \\
\hline . & $\underset{\Xi}{\Xi}$ & 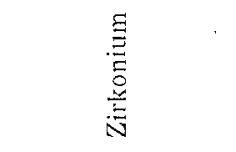 & $\stackrel{\vec{j}}{3}$ \\
\hline
\end{tabular}


Zur Kenntniss einiger seltener Erden.

699

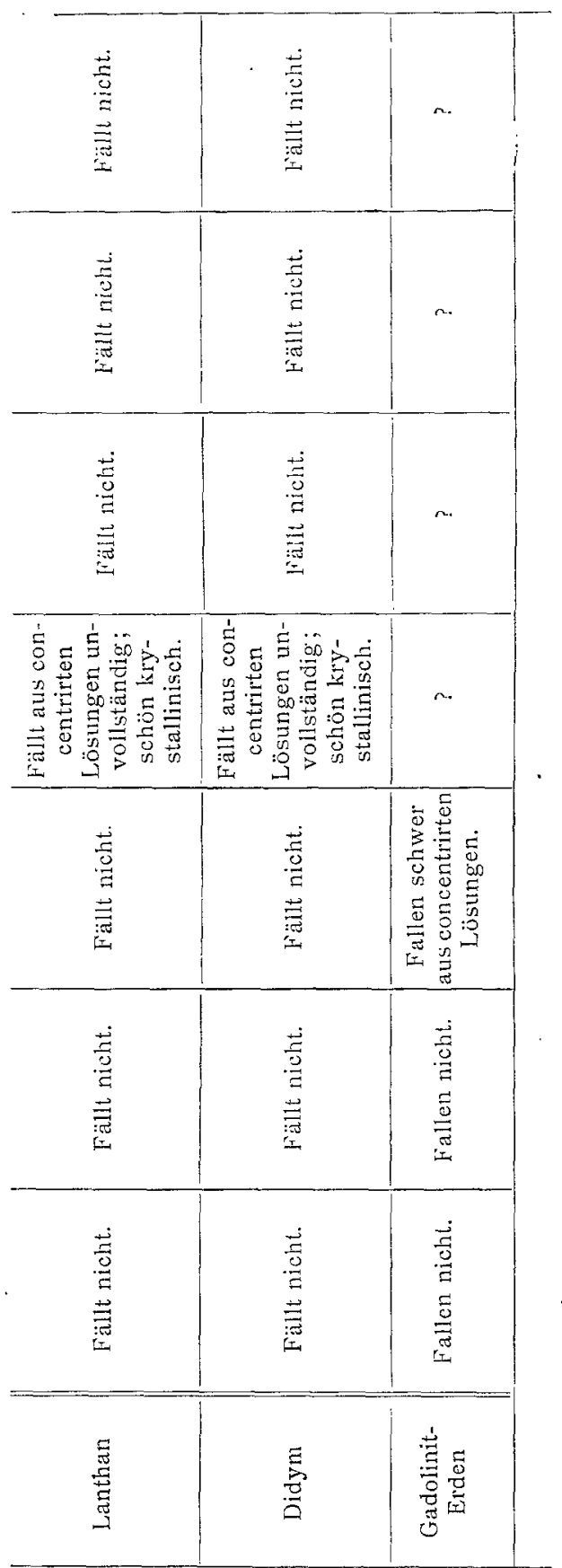

